\title{
CYCLOADDITION REACTIONS OF CARBONYL COMPOUNDS POSSESSING HIGH ENERGY CONTENT
}

\author{
Nicholas J. TURRO \\ Department of Chemistry, Columbia University, \\ New York 10027, USA
}

\begin{abstract}
Detailed structure and kinetic analyses of the photocycloaddition of alkanones to (a) $\alpha, \beta$-unsaturated nitriles and (b) enol ethers have been performed. The excited singlet states of alkanones were found to add stereospecifically and regiospecifically to $\alpha, \beta$-unsaturated nitriles. Alkanone triplets do not add to unsaturated nitriles but instead transfer triplet excitation (at the diffusion controlled rate) and thereby sensitize dimerization and cis-trans isomerization of the nitrile. Even when all the alkanone singlets are quenched by a ground state nitrile, oxetane formation is relatively inefficient. The possibility is put forward that singlet exciplex of alkanone and nitrile is formed directly, but then dissociates (undergoes internal conversion) about ten times faster than it collapses to oxetane.

In contrast, both $S_{1}$ and $T_{1}$ of alkanones add to enol ethers and effect both oxetane formation and cis-trans isomerization. Evidence is produced in support of singlet and triplet biradicals as intermediates to oxetanes. The possibility of a precursor to the biradicals is considered.

Alkanone fluorescence quenching, by ethylenes known to form oxetanes, is used as a probe of the stereoelectronic requirements of the quenching process and to study excited state conformations.
\end{abstract}

\section{INTRODUCTION}

In 1909, Paterno and Chieffi reported that after exposure of a solution of benzophenone in 2-methyl-2-butene to the brilliant and reliable sunlight of Italy for a few days, they were able to isolate, from the irradiated solution, a 1:1 adduct of benzophenone and 2-methyl-2-butene for which the oxetane structure (I) was proposed ${ }^{1}$. Hardly any further work on this interesting reaction was reported until 1954 when Büchi ${ }^{2}$ showed that the PaternoChieffi structure I was correct. No mechanistic studies of this reaction were reported until 1964 when stimulating papers appeared by Arnold, Hinman and Glick ${ }^{3}$ and Yang ${ }^{4}$ and his collaborators. These papers, coupled with the flourishing of new theoretical concepts and new techniques for the study of photochemical mechanisms, galvanized a number of important studies into the details of photochemical oxetane formation. Two extensive recent reviews attest to the synthetic usefulness and intriguing mechanisms of these reactions ${ }^{5,6}$. Much of the mechanistic work on photochemical oxetane formation deals with the addition of aromatic ketones to ethylenes. In this 
paper we shall be mainly concerned with the photocycloaddition of alkyl ketones to ethylenes ${ }^{7-14}$.

$$
\left(\mathrm{C}_{6} \mathrm{H}_{5}\right)_{2} \mathrm{CO}+\left(\mathrm{CH}_{3}\right)_{2} \mathrm{C}=\mathrm{CHCH}_{3} \stackrel{h v}{\longrightarrow} \underbrace{\mathrm{O}-\mathrm{C}_{6} \mathrm{H}_{5}}_{\mathrm{I}}
$$

\section{CHARACTERISTIC SPECTROSCOPIC PROPERTIES AND PHOTOREACTIONS OF ALKANONES}

Solutions of alkanones, in u.v. transparent organic solvents, generally display a weak extinction $\left(\varepsilon_{\max .} \sim 10\right.$ to 30$)$, relatively broad absorption band which maximizes in the region of 280 to $320 \mathrm{~nm}^{15}$. It has been demonstrated that this absorption band results from photon induced excitation (Figure 1) of an electron from a non-bonding $(n)$ orbital (which is essentially

Configurations, States, Transitions
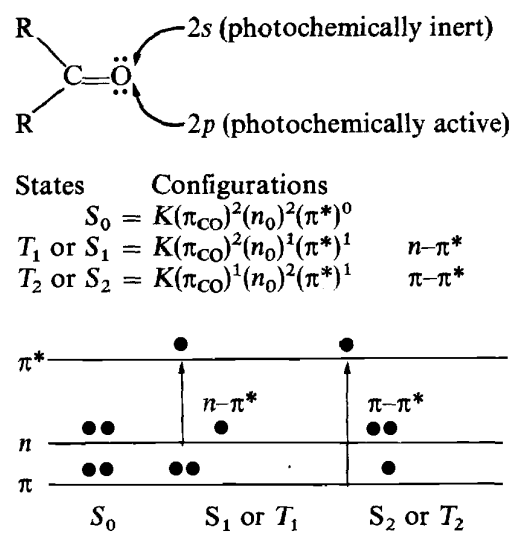

Figure 1. One electron orbital description of an $n \rightarrow \pi^{*}$ and $\pi \rightarrow \pi^{*}$ excitation. In the approximation electron-electron repulsions (which are expected to be a main source of any chemical differences between $S_{1}$ and $T_{1}$ ) are ignored; therefore, it is not capable, without appropriate modification, of providing a basis for distinctions in the chemical properties of $S_{1}$ and $T_{1}$

localized on the carbonyl oxygen atom and which is located in the plane of the carbonyl function) to an unoccupied anti-bonding $\pi^{*}$ orbital (which is delocalized over the carbon and oxygen atoms of the carbonyl group and which is located in the space above and below the plane of the carbonyl function). This $n \rightarrow \pi^{*}$ electron excitation occurs without 'spin flip' so that an $n, \pi^{*}$ singlet (spin paired) electronically excited state is the first species produced when alkanones absorb a photon of u.v. light of wavelength 280 to $320 \mathrm{~nm}$. We shall be concerned, in this manuscript, with the chemical nature, chemical dynamics and the fate of the $n, \pi^{*}$ singlet state subsequent to its formation. 
In general, the initially formed ketone $n, \pi^{*}$ singlet state $\left(K_{1}^{*}\right) \dagger$ will become thermally equilibrated at a rate which is much faster than any chemical process. Thus $K_{1}$ is the first chemically interceptable intermediate produced after light absorption. Three chemical ( $a-$ hydrogen abstraction; $b-\alpha$ cleavage ; $c-$ cycloaddition to ethylenes) and three physical ( $d$ - fluorescence ; e - intersystem crossing to the triplet $n, \pi^{*}$ state; $\mathrm{f}$ - electronic energy transfer to a qualified acceptor) will, usually, be the only important paths for deactivation of $K_{1}$. Appropriate choice of reaction conditions will eliminate possibilities $\mathrm{a}, \mathrm{b}$ and $\mathrm{f}$, so that one can study the cycloaddition of $K_{1}$ to ethylenes and its competition with deactivation of $K_{1}$ via fluorescence (to yield $S_{0}$ and a photon) and intersystem crossing (to yield $T_{1}$, the $n, \pi^{*}$ triplet state). Many alkanones ${ }^{16}$ in the absence of external quenchers undergo efficient intersystem crossing to yield $T_{1}$. In the case of acetone ${ }^{17}$, the quantum yield $\ddagger$ of triplets is $1.0 \pm 0.1$. This means that unless the $S_{1}$ state of acetone is intercepted by an external reagent, then $T_{1}$ will be produced quantitatively.

\section{ENERGETICS AND DYNAMICS OF ALKANONE $S_{1}$ AND $T_{1}$ STATES}

Figure 2 is an energy diagram which summarizes the chemically significant processes which interconnect $S_{0}, S_{1}$ and $T_{1}$ of acetone. Such a diagram provides a concise and informative summary of the energetics and dynamics which will be crucial in understanding the photocycloaddition reactions of alkanones. Excitation of acetone with light of wavelength 260-300 nm (after vibrational relaxation) produces $S_{1}$, the $n, \pi^{*}$ singlet state, in quantitative yield. The important features of $S_{1}$ for acetone are: (1) the fact that it possesses $\sim 84 \mathrm{kcal} / \mathrm{mole}$ excess electronic energy over $S_{0}$, acetone's ground state; (2) in the absence of external quenchers of $S_{1}$, only two processes deactivate $S_{1}$ : (a) intersystem crossing to $T_{1}$ and (b) fluorescence to yield $S_{0}$; (3) the rate constant for intersystem crossing $\left(k_{S T}\right)$ is $5 \times 10^{8} \mathrm{sec}^{-1}$ while the rate constant for fluorescence $\left(k_{F}\right)$ is only $10^{6} \mathrm{sec}^{-1}$; (4) both $S_{1}$ and $T_{1}$ are $n, \pi^{*}$ states.

Since the lifetime $\left(\tau_{S}\right)$ of $S_{1}$ is defined as the reciprocal of the rates of deactivation of $S_{1}$ (equation 1), we see that the lifetime of $S_{1}$ is inherently limited by the rate of intersystem crossing. Although the quantum yield of

$$
\tau_{S} \equiv\left(k_{F}+k_{S T}\right)^{-1} \sim k_{S T}
$$

fluorescence from $S_{1}$ for acetone is quite low ( $\sim 0.01$ to 0.001$)$, this emission is easily detected by sensitive fluorescence techniques. As we shall see,

$\dagger$ An asterisk superior to a state symbol is intended to imply excess vibrational excitation. Absence of a superior asterisk implies thermal equilibrium of the corresponding state. Inferior 1 is intended to imply an excited singlet state, inferior 3 is intended to imply a triplet state and inferior 0 is intended to imply a ground electronic state.

$¥$ The quantum yield is a measure of the efficiency and not the rate of a photochemical process. Quantum yields are defined as the number of molecules undergoing the process of interest divided by the number of photons absorbed by the system, i.e. output over input, a dimensionless term. In general, the quantum yield measures the ratio of the rate of the process of interest divided by the sum of all rates deactivating the reactive state. The yield of the reactive state must also be known to define properly the rate ratio:reaction/sum of all deactivation. 
NICHOLAS J. TURRO

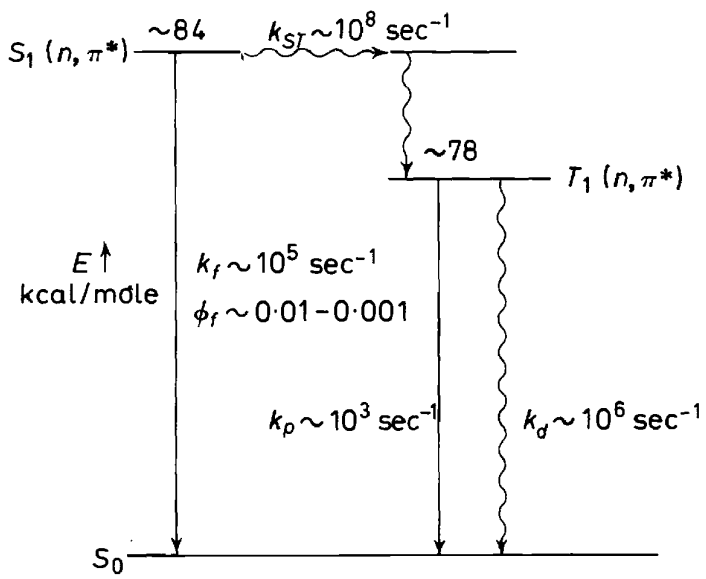

Figure 2. Energy diagram for acetone in solution at $25^{\circ} \mathrm{C}^{15}$. Kasha's rule states that in the absence of evidence to the contrary, it can be assumed that the solution photochemistry of organic molecules is initiated in either $S_{1}$ or $T_{1}$. Thus, we shall assume, since there is good evidence for the validity of Kasha's rule, that only $S_{1}$ and $T_{1}$ of alkanones need be considered, and that vibrationally 'hot' electronically excited states are of no importance in the systems under discussion

fluorescence will be a particularly versatile and valuable probe for quantitative study of the photocycloaddition of $S_{1}$ of alkanones to ethylenes.

If $T_{1}$ is produced, its major mode of deactivation is intersystem crossing to $S_{0}{ }^{17}$. It should be noted that the latter process has a rate constant $\left(k_{\mathrm{D}}\right)$ which is considerably smaller $\left(\sim 10^{6} \mathrm{sec}^{-1}\right)$ than the intersystem crossing process $\left(k_{S T} \sim 5 \times 10^{8} \mathrm{sec}^{-1}\right)$ which serves as the major deactivation path for $S_{1}$. The lifetime of $T_{1}\left(\tau_{T}\right)$ is thus given by equation 2 .

$$
\tau_{T}=\left(k_{D}\right)^{-1}
$$

Although the $T_{1}$ state possesses about the same amount of excess electronic energy ( $\sim 78 \mathrm{kcal} / \mathrm{mole})$, we shall see that quenching by electronic energy transfer, to a qualified energy acceptor, is generally more important for $T_{1}$ than for $S_{1}$.

\section{THE CHEMICAL NATURE OF ALKANONE $S_{1}$ AND $T_{1}$ STATES}

To a first approximation, let us consider the electronic distribution of both the $S_{1}$ and $T_{1}$ states of alkanones as that expected in terms of $n, \pi^{*}$ states which contain the usual core electrons of the ground state carbonyl function, excepting that the highest energy $n$ orbital and the lowest energy $\pi^{*}$ orbital are both half occupied ${ }^{18}$.

Although such an approach may seem to be simplistic to the point of naiveté, we shall see that in spite of some inherent but recognizable deficiencies this model provides a powerful qualitative basis for the interpretation of the photocycloaddition of alkanones to ethylenes. 


\section{CYCLOADDITION REACTIONS OF CARBONYL COMPOUNDS}

Two important features of $S_{1}$ and $T_{1}$ which this model is unable to handle at all are:(1) the magnitude of excess electronic energy (which can fortunately be experimentally determined) and electronic distribution for $S_{1}$ and $T_{1}$; (2) the nuclear geometries of $S_{1}$ and $T_{1}$.

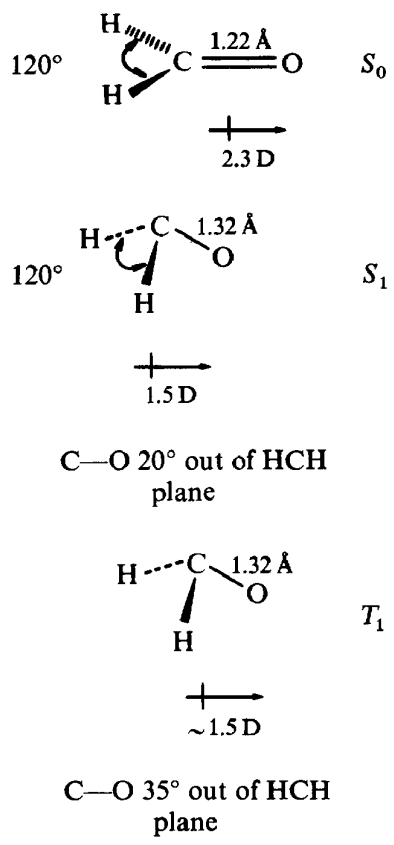

Figure 3. Structural parameters of formaldehyde in its $S_{1}, T_{1}$ and $S_{0}$ states. The $S_{1}$ and $T_{1}$ states are 75 to $80 \mathrm{kcal} / \mathrm{mole}$ higher in energy than $S_{0}$. Only a few hundred reciprocal centimetres of energy are required to convert one pyrimidal form of the $S_{1}$ or $T_{1}$ states into its mirror image. Thus, both of these excited states are rapidly inverting species

In order to obtain a feeling for points (1) and (2) above, consider Figure 3 which summarizes the nature of the formaldehyde molecule in its $S_{1}\left(n, \pi^{*}\right)$ and $T_{1}\left(n, \pi^{*}\right)$ states. We see that formaldehyde exists in a puckered, rapidly inverting shape in both $S_{1}$ and $T_{1}$, with the latter state being somewhat more strongly puckered ${ }^{15}$. Although the dipole moments of $S_{1}$ and $T_{1}$ have decreased, relative to $S_{0}$, (1) the negative end of the excited state dipole is still toward the carbonyl oxygen atom and (2) the decrease in dipole moment is consistent with the expected charge redistribution associated with excitation of an $n$ electron (essentially localized on the carbonyl oxygen atom) into a $\pi^{*}$ orbital (delocalized over both atoms of the carbonyl function).

We can now summarize the model for $S_{1}$ and $T_{1}$ by Figure 4 . With the caveat that the $\mathrm{C}-\mathrm{CO}-\mathrm{C}$ system may be somewhat non-planar in an $n, \pi^{*}$ state, we can predict on the basis of Figure 4 that: (1) $S_{1}$ and $T_{1}$ of alkanones are 'bipolar' intermediates; (2) $S_{1}$ and $T_{1}$ are electrophilic and radical-like and have as their main source of electron deficiency the half-filled $n$ orbital (a 'positive hole') roughly in the plane of the $\mathrm{C}-\mathrm{CO}-\mathrm{C}$ group and close to 
the oxygen end of the electronically excited carbonyl function; (3) $S_{1}$ and $T_{1}$ are nucleophilic (and possibly radical-like) in the area of space roughly above and below the electronically excited $\mathrm{C}=\mathrm{O}$ function as the result of the presence of a $\pi^{*}$ electron.

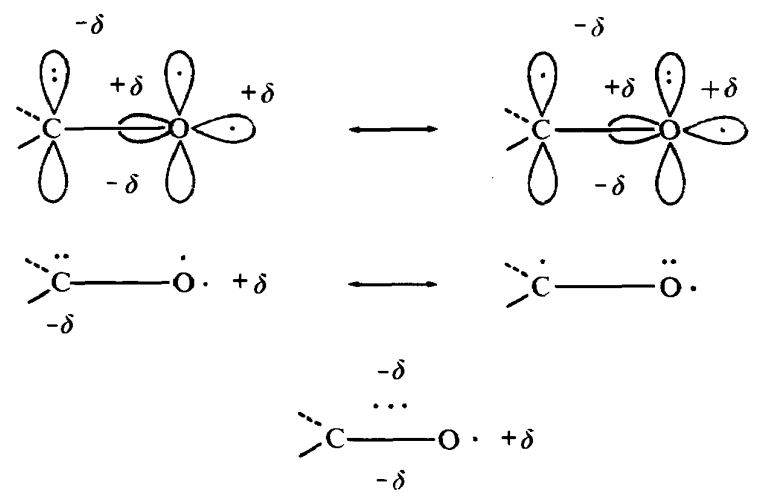

Figure 4. A primitive model for the $n, \pi^{*} S_{1}$ and $T_{1}$ states for an alkanone

A legitimate test of the validity and usefulness of this model resides in its ability to rationalize and predict variations in the photochemical cycloaddition of $S_{1}$ and $T_{1}$ of alkanones to ethylenes as a function of ketone and ethylene structure.

We are now hopefully armed with sufficient background with which to confront the available data of photocycloadditions of alkanones to ethylenes. We shall consider the photocyloaddition of alkanones to $\alpha, \beta$-unsaturated nitriles and enol ethers, their stereochemical course, their regiospecficity and their structure/reactivity relationships.

\section{PHOTOCYCLOADDITION OF ACETONE TO ETHYLENES}

In the following discussion we shall use acetone as our model alkanone from which to derive our understanding of the photocycloaddition of $S_{1}$ and $T_{1}$ to ethylenes. Table 1 and Table 2 summarize some of the reported photocycloadditions of acetone to some $\alpha, \beta$-unsaturated nitriles and some enol ethers.

\section{STEREOSPECIFICITY AND REGIOSPECIFICITY OF THE PHOTOCYCLOADDITION OF ACETONE TO ETHYLENES}

Let us consider the stereospecificity and regiospecificity of the photocycloaddition of acetone with (1) unsaturated nitriles and (2) enol ethers. Although good yields of oxetanes are generally observed, there are striking differences in the stereochemical courses followed by these two classes of ethylenes. Thus, the photocycloaddition of acetone to acrylonitrile, methacrylonitrile and crotononitrile ${ }^{19}$ are highly regiospecific and the photocycloaddition of acetone to both cis- and trans-1,2-dicyanoethylene (c-DCE and $t$-DCE, respectively) is highly stereospecific $c^{7,8,13}$. On the other hand, the 


\section{CYCLOADDITION REACTIONS OF CARBONYL COMPOUNDS}

corresponding reaction of acetone to enol ethers is only modestly regioselective ${ }^{20-24}$ at best, and addition to cis- and trans-1-methoxybutene ( $c$-MB and $t$-MB, respectively) is not stereospecific. It is also important to note that the most significant side reaction with both DCE and MB is cis-trans

Table 1. Photocycloaddition of acetone to $\alpha, \beta$-unsaturated nitriles

trans- $\mathrm{NCCH}_{\text {trans }-\mathrm{CH}{ }_{3} \mathrm{CH}=\mathrm{CHCN}}$

- No 3-cyanooxetane observed.

b Mixture of cis- and trans-isomers.

c Only stereoisomer formed at low conversions.

isomerization. It is also significant that although for acrylonitrile and methacrylonitrile, ethylene dimerization is an important side reaction, dimers have not generally been isolated as significant products with enol ethers $^{25}$. A final point worthy of mention is the fact that there appear to be no significant solvent effects on the regioselectivity of the photocycloaddition of acetone to enol ethers ${ }^{26}$.

At this point some general conclusions about the possible mechanism(s) for photocycloaddition of acetone to unsaturated nitriles and enol ethers are 
NICHOLAS J. TURRO

Table 2.'Photocycloaddition of acetone to enol ethers

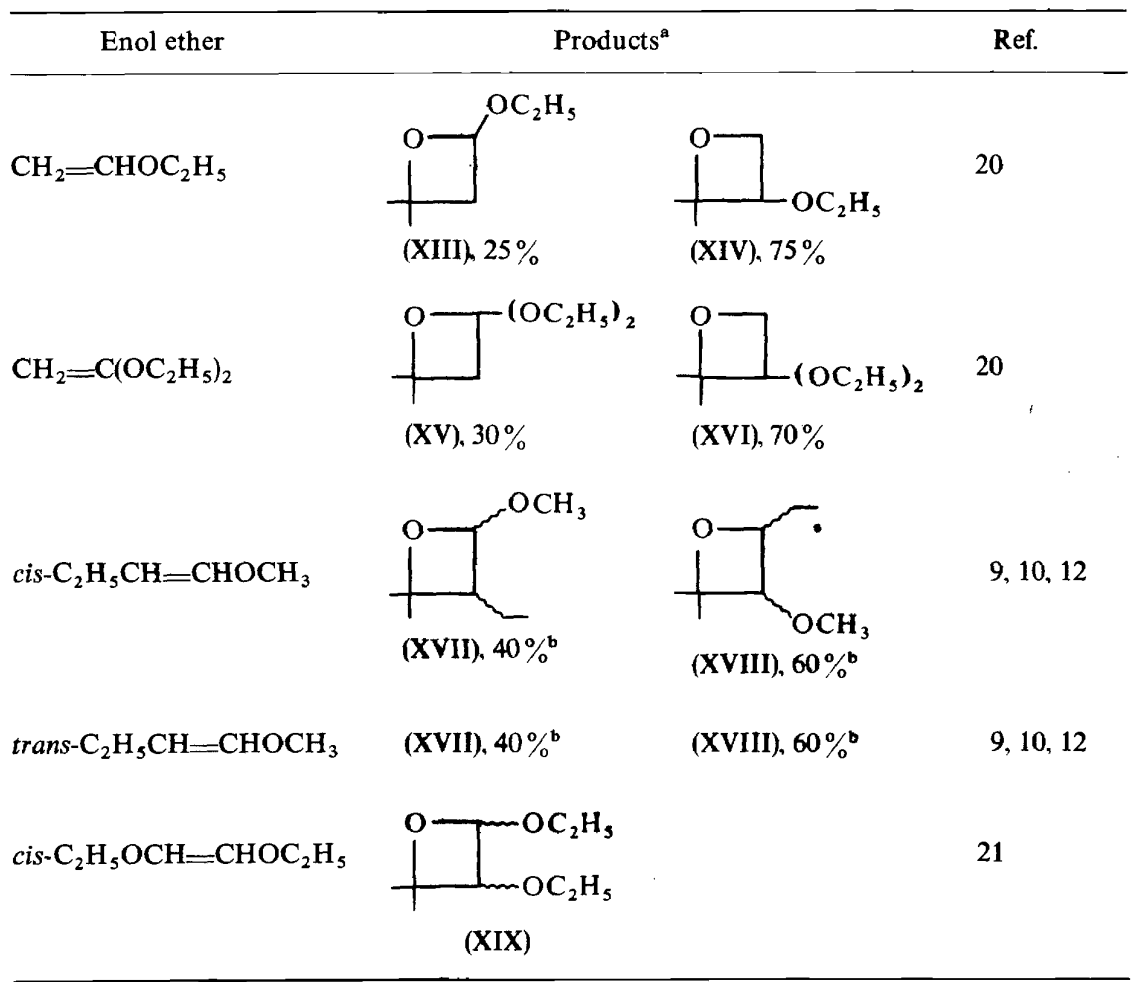

- Yields in this table are relative only. Chemical yields are greater than 60 per cent.

- Mixture of stereoisomers. Stereospecificity depends on the enol ether concentration. cis-trans-Isomerization of the enol ether also occurs.

possible: the photocycloaddition of excited acetone to unsaturated nitriles possesses a necessary (but not sufficient) characteristic of concerted reactions-stereospecificity ${ }^{27}$. The regioselectivity suggests a transition state in which the excited carbonyl carbon is negatively polarized and the carbonyl oxygen is positively polarized. The photocycloaddition of excited acetone to enol ethers, however, is almost certainly not concerted and is consistent with the intervention of some intermediate species of sufficiently long life or sufficiently rapid $\mathrm{C}-\mathrm{C}$ bond rotations to lose initial ethylene stereochemistry. However, intermediate bipolar species such as (XXa) (Figure 5) are probably

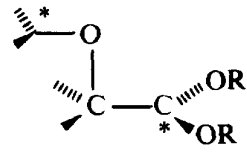

$\mathrm{XXa}$

$$
\text { * }=+,- \text { or } \text {. }
$$

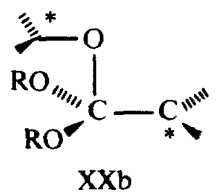

Figure 5. Possible 1,4-bifunctional intermediates from addition of $S_{1}$ or $T_{1}$ of an alkanone to an enol ether 


\section{CYCLOADDITION REACTIONS OF CARBONYL COMPOUNDS}

ruled out on the basis of (1) poor regiospecificity [form (XXa) should predominate strongly relative to $(\mathrm{XXb})$, and therefore unsymmetrically substituted enol ethers should yield essentially only one oxetane], and (2) lack of solvent effect on regiospecificity.

Arguments such as the above, which are based mainly on the basis of the structure of products, are generally not very compelling. With photocycloadditions they are particularly unsatisfactory. Questions which are unanswered, but which are amenable to experimental probing, are:(1) are the dimerizations and cis-trans isomerizations (with unsaturated nitriles) associated with photocycloaddition or are they unrelated side reactions? (2) is the cis-trans isomerization (with enol ethers) associated with photocycloaddition or is it an unrelated side reaction? (3) which excited state(s) of the ketone is (are) involved in (a) photocycloaddition, (b) cis-transisomerization and dimerization? (4) does an excited ethylene (produced by photosensitization) ever cycloadd to a ground state ketone? (5) are intermediates other than $S_{1}$ and $T_{1}$ of the ketones involved? (6) why do excited alkanones appear to cycloadd to electron-poor ethylenes and electron-rich ethylenes with comparable facility?

To answer the above and many other related mechanistic questions we must make use of photochemical dynamics of alkanone $S_{1}$ and $T_{1}$ states (Figure 2) and structural excited state reactivity relationships.

\section{PHOTOCYCLOADDITION OF ALKANONES TO UNSATURATED NITRILES}

First let us consider the question: Is the $S_{1}$ or $T_{1}$ (or both) state of the unsaturated nitrile involved in the photocycloaddition? We can confidently answer this question in the negative. The $S_{1}$ state is an energetically unattainable intermediate when light of $310 \mathrm{~nm}$ is used to promote photocycloaddition, and can therefore be ruled out. The $T_{1}$ state of unsaturated nitriles ${ }^{28-30}$ probably lies $<65 \mathrm{kcal} /$ mole above its ground state (Figure 6). It is therefore possible selectively to excite the nitrile triplet with a number of sensitizers which (a) absorb all of the exciting light, (b) are incapable of exciting either $S_{1}$ or $T_{1}$ of the alkanone and (c) efficiently and selectively excite the nitrile triplet. Thus, one can irradiate a solution containing such a sensitizer, acetone and an unsaturated nitrile. The results are convincing: no oxetanes are formed ${ }^{13,19}$, but only cis-trans-isomerization ${ }^{13}$ and/or dimerization $^{19}$, reactions known to be characteristic of nitrile triplet $^{28-30}$, are observed.

Having eliminated $S_{1}$ and $T_{1}$ of the unsaturated nitrile as plausible reaction intermediates, let us now consider the question: Is the alkanone $S_{1}$ or $T_{1}$ state (or both) involved in the photocycloaddition? In this case we can find both direct and indirect experimental evidence that provides powerful arguments for the conclusion that only the $S_{1}$ state of the alkanone is involved in the photocycloaddition. The evidence is as follows: (a) the quenching of alkanone singlets by nitrile follows Stern-Volmer kinetics:

$$
\phi_{F}^{0} / \phi_{F}=1+k_{q}^{F} \tau_{S}[\mathrm{RCN}]
$$




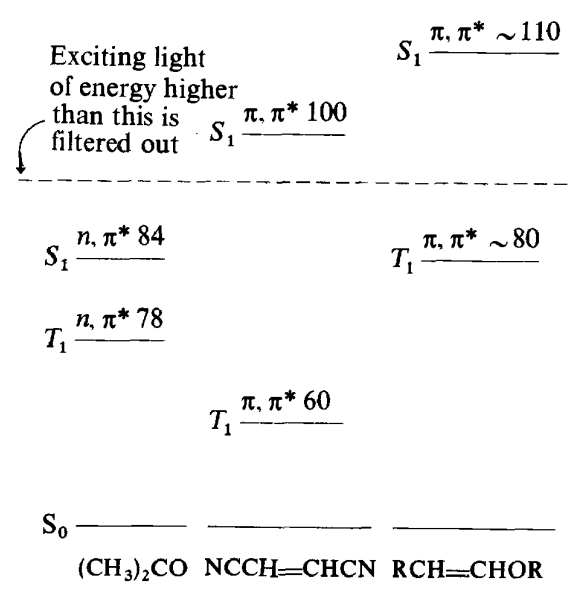

Figure 6. Energy levels of the $S_{1}$ and $T_{1}$ states of acetone, 1,2-dicyanoethylene and an enol ether. Kasha's rule (Figure 2) allows us to consider only the $S_{1}$ and $T_{1}$ states of the above molecules as significant to their solution phase photochemistry. The most convenient experimental test for the intermediacy of $S_{1}$ is the observation of fluorescence. The most convenient experimental test for the intermediacy of $T_{1}$ of alkanone is the observation of quenching of the photochemical reaction of interest by 1,3-pentadiene

where $\phi_{F}^{0}$ is the quantum yield for alkanone fluorescence in the absence of nitrile, $\phi_{F}$ is the quantum yield for alkanone fluorescence in the presence of nitrile of concentration [RCN], $k_{q}^{F}$ is the bimolecular rate constant for fluorescence quenching by nitrile and $\tau_{s}$ is the lifetime of fluorescent alkanone in the absence of nitrile. When a photoreaction follows equation 3 , we have prima facie evidence that bimolecular quenching is competing with unimolecular or pseudo-unimolecular processes for deactivation of alkanone $S_{1}$ states. Fluorescence quenching is a very convenient technique and is quite specific. In effect we are using a photon to simultaneously 'label' and activate an alkanone and we have a built-in 'clock', the fluorescent lifetime, $\tau_{\boldsymbol{S}}$, to measure how fast our labelled ketone reacts.

(b) Alternatively, the kinetic expression

$$
\left(1 / \phi_{\mathrm{ox}}\right)=(1 / a)\left\{1+1 / k_{q}^{\mathrm{ox}} \tau_{S}[\mathrm{RCN}]\right\}
$$

would be expected if the oxetane formation occurred only from $S_{1}$. In this equation $\phi_{\mathrm{ox}}$ is the quantum yield for oxetane formation, $a$ is the fraction of $S_{1}$ states that form oxetane molecules at a concentration of nitrile equal to [RCN], $k_{q}^{\text {ox }}$ is the bimolecular rate constant for quenching of the excited singlet precursor to oxetane and $\tau_{S}$ is the lifetime of the $S_{1}$ alkanone in the absence of nitrile.

Since both equations 3 and 4 are experimentally found to be linear, if the alkanone singlet state is a precursor to oxetane formation then $k_{q}^{\mathbf{o x}} \tau_{S}$ from equation 4 should equal $k_{q}^{F} \tau_{s}$ from equation 3 . In fact for $t$-DCE, $k_{q}^{\text {ox }}=5.1$ and $k_{q}^{F} \tau_{s}=6.7$, which are very close to the same value within the experimental error of about \pm 50 per cent ${ }^{13}$. Thus, direct kinetic analysis is consistent with 
$S_{1}$ as the only state involved in photocycloaddition to $t$-DCE. Similar results obtain for other nitriles and acetone.

(c) Further support for the absence of $T_{1}$ of alkanones as precursors of the photocycloaddition is the fact that 1,3-pentadiene, a compound known to quench acetone triplets at the diffusion controlled rate ${ }^{31}$, does not quench the photocycloaddition of acetone to unsaturated nitriles ${ }^{13,19}$, but strongly inhibits nitrile cis-trans-isomerization ${ }^{13}$ and/or dimerization ${ }^{19}$. In fact, no cis-trans-isomerization appears to be sensitized by acetone singlets, thus ruling out an energetically feasible but spin forbidden singlet alkanone to triplet ethylene excitation transfer ${ }^{32}$. Thus, we can conclude that alkanone $S_{1}$ states add to unsaturated nitriles and alkanone $T_{1}$ states sensitize nitrile cis-trans-isomerization and/or dimerization. The latter sensitization is almost certainly via triplet energy transfer from the alkanone $T_{1}$ state to produce the nitrile $T_{1}$ state ${ }^{28-30}$. The conclusion that only alkanone $S_{1}$ states add to unsaturated nitriles is also consistent with the stereospecificity of the photocycloaddition of acetone to cis- and trans-1,2-dicyanoethylene and to cisand trans-crotononitrile ${ }^{19}$, and with the failure of aryl ketones (which possess $S_{1}$ states so short-lived that their involvement in bimolecular processes is very unlikely) to add to unsaturated nitriles ${ }^{13}$.

Let us now ask the question: When a nitrile quenches an alkanone $S_{1}$ state, does an oxetane form directly or does an intermediate occur? The problem of deciding whether two newly forming $\sigma$ bonds are closed simultaneously or whether they close one after the other is very often at the heart of the discussion of cycloaddition mechanisms ${ }^{27}$. Conceptually, we must consider as mandatory that an elementary chemical step is concerned if the reaction profile, connecting the starting reagents with the products, contains only a single activation peak. It is generally assumed that such a concerted path is required to lead to stereospecific reaction. Since intermediates are excluded essentially by definition, in a one-step concerted reaction, 'proof' of mechanism, in such a case, must rely on 'exclusion of all alternate plausible and conceivable mechanisms', i.e. indirect proof is required. One can imagine the occurrence of cycloaddition reactions which proceed via characterizable or trappable intermediates which are nonetheless stereospecific because both advance (cycloaddition) and retrograde (dissociation) motions, by the intermediate, along the reaction coordinate require a lower activation energy than configurational scrambling of the intermediate. Quite often it is the rotations about an essential single bond in a bifunction intermediate (e.g. 1,4-dipole or a 1,4-biradical) which compete with collapse of the intermediate to products. There is really no guarantee that the rates of rotations about a 'single' bond in such an intermediate will be commensurate with those of a stable molecule possessing no free valences. It is entirely possible that although the free valence centres in an intermediate may be bonded (perhaps feebly or comparably to the first formed bond) from the beginning of an activation step, various paths in addition to cycloaddition or reversion for diverting, or perhaps even intercepting the intermediate may exist. We know from equation 4 that the efficiency of oxetane formation is given by $a$, which equals $\frac{1}{13}$ for $t$-DCE and $\frac{1}{12}$ for $c$-DCE. In other words, both reactions are relatively inefficient ${ }^{13}$. Furthermore, since cis-trans-isomerization is not singlet sensitized, the quenched acetone singlets and quenching nitrile must 
also have a path to return to their original ground states after degrading electronic excitation ${ }^{33}$. Thus, we can conclude that either (a) oxetane formation and some other independent process for quenching alkanone $S_{1}$ occurs simultaneously and competitively (e.g. it is possible that collisions between $S_{1}$ and nitrile will occur and a bond is formed but orientational problems or unfavourable electronic factors slow down the rate of formation of the second bond relative to reversal to starting materials) or (b) DCE quenches $S_{1}$ to form an intermediate which can then partition itself between oxetane formation and collapse to ground state acetone and ground state DCE. Since $t$-DCE and $c$-DCE are found not to isomerize after quenching an $S_{1}$ singlet, any intermediate must be capable of collapsing facilely and

Table 3. Relative quenching rates of alkanone fluorescence by $\alpha, \beta$-unsaturated nitriles ${ }^{a}$

\begin{tabular}{ccccc}
\hline Alkanone & trans- $\mathrm{NCCH}=\mathrm{CHCN}$ & $\mathrm{CH}_{2}=\mathrm{CHCN}$ & $\mathrm{CH}_{2}=\mathrm{C}_{\left(\mathrm{CH}_{3}\right) \mathrm{CN}}$ & $\mathrm{CH}_{3} \mathrm{CH}=\mathrm{CHCN}$ \\
\hline & $1.0^{\mathrm{b}}$ & 0.046 & 0.020 & $<0.01$ \\
& & & & \\
\hline & & & & \\
\hline
\end{tabular}

a Acetonitrile solution.

b The absolute quenching rate constant is $2.5 \times 10^{9} \mathrm{M}^{-1} \mathrm{sec}^{-1}$.

c The absolute quenching rate constant is $5.0 \times 10^{9} \mathrm{M}^{-1} \mathrm{sec}^{-1}$.

stereospecifically to oxetane or starting materials. What kind of plausible intermediate could possess such properties? Let us consider the relative reactivity of quenching of alkanone fluorescence by unsaturated nitriles (Table 3). It can be seen that the quenching of fluorescence (and presumably the formation of oxetane) supports the proposition that the $S_{1}$ alkanone behaves as a nucleophile, since the rate of quenching increases with decreasing $\pi^{*}$ energy of the $\mathrm{C}=\mathrm{C}$ bond (Figure 7 ). It should also be noted that in spite of its increased steric disadvantage, $t$-DCE is a far better quencher than acrylonitrile or methacrylonitrile. These data, when coupled with (a) the reaction stereospecificity and (b) the regiospecificity of reaction (which requires a bond between the carbonyl oxygen and the cyanide-bearing carbon of the ethylene) ${ }^{34}$ suggests to us that neither biradical intermediates nor bipolar intermediates possessing one fully formed $\sigma$ bond are involved. On the other hand, an excited state complex (generally called an exciplex ${ }^{35}$ ) possessing some charge transfer character (Figure 9) is an attractive intermediate to explain (a) the inefficiency of oxetane formation; (b) the relative reactivities listed in Table 3; (c) the stereospecificity of oxetane formation and reversal to starting materials; (d) the lack of cis-trans-isomerization of nitrile and (e) the regiospecificity of oxetane formation. MO calculations (Figure 7) show that if a bipolar intermediate were operating, then acrylonitrile (which also possesses less steric hindrance to reaction) and a more positive $(\beta)$ carbon atom for attack and a more negative $(\alpha)$ carbon atom for attack should quench $S_{1}$ alkanones faster than $t$-DCE. On the other hand, 


\section{CYCLOADDITION REACTIONS OF CARBONYL COMPOUNDS}

$$
\begin{aligned}
& +\begin{array}{ccc}
.0 .11 \quad-0.016 & E_{\pi^{*}} \\
= & \alpha N & \alpha-0.5 \beta
\end{array} \\
& +0.18-0.024 \\
& =\int_{\mathrm{CN}}^{\mathrm{CN}} \alpha-0.4 \beta \\
& \underbrace{\mathrm{NC}}_{\mathrm{CN}} \alpha-0.24 \beta
\end{aligned}
$$

Figure 7. Energy of the lowest unoccupied orbitals and charge distributions of some $\alpha, \beta$ unsaturated nitriles
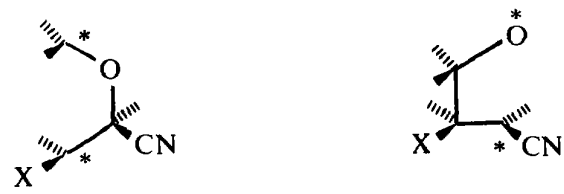

$$
\begin{aligned}
& \mathrm{X}=\mathrm{CH}_{3}, \mathrm{H}, \mathrm{CN} \\
& *=+,- \text { or } .
\end{aligned}
$$

Figure 8. Possible 1,4-bifunctional intermediates from the addition of $S_{1}$ of acetone to an $\alpha, \beta$-unsaturated nitrile
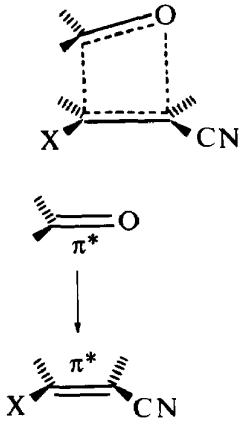
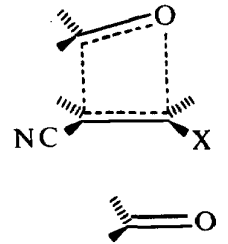

$\equiv$

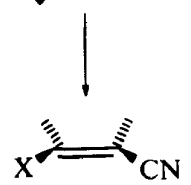

Exciplex

Figure 9. Possible transition states for the photocycloaddition of $S_{1}$ of alkanones to $\alpha, \beta$ unsaturated nitrile. The exciplex is envisioned as achieving stabilization via charge transfer interactions with the $S_{1} \pi^{*}$ electron being 'donated' to the vacant $\pi^{*}$ orbital of the nitrile 
$t$-DCE possesses a lower vacant $\pi^{*}$ orbital than acrylonitrile, a situation precisely consonant with charge transfer interaction from $S_{1}$ to the nitrile $\pi^{*}$ orbital (Figure 9 ). We thus imagine this stabilizing charge transfer interaction which causes the faster rate of quenching for $t$-DCE, will stabilize an exciplex of excited alkanone and nitrile. There is good analogy in the literature for low activation for formation and dissociation of (ground state) charge transfer complexes ${ }^{35}$, which can then form $\sigma$ bonds with low activation energy. These processes should be just as, or more, facile in an exciplex.

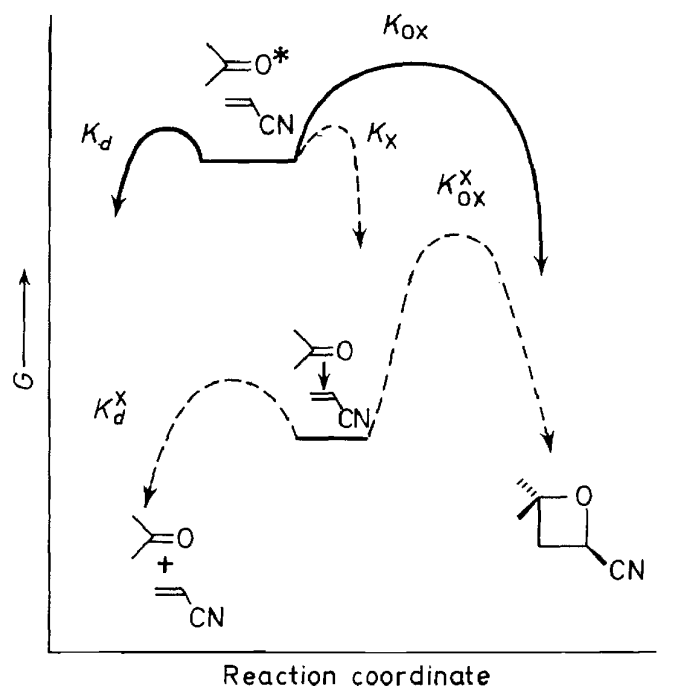

Figure 10. Possible reaction profiles for the photocycloaddition of an $S_{1}$ alkanone to an $\alpha, \beta$-unsaturated nitrile. Since $S_{1}$ (a) possesses two half-vacant orbitals which are in approximately orthogonal planes and (b) may be somewhat non-planar about the $\mathrm{C}-\mathrm{CO}-\mathrm{C}$ group, orbital symmetry rules cannot provide a firm basis for expecting concerted or multi-step oxetane formation from $S_{1}$ and nitrile directly, or from the exciplex: R. B. Woodward and R. Hoffmann, Angew. Chem. Internat. Ed. Engl. 8, 781 (1969). Perturbational MO calculations by Professor W. C. Herndon (Texas Tech) indicate that $S_{1}$ and DCE can form oxetane via a low energy, concerted path. Consideration of highest occupied and lowest unoccupied orbitals leads to a similar conclusion: G. Ahlgren and B. Akermark, Tetrahedron Letters, 1885 (1970)

The mechanism for photocycloaddition of alkanones to nitriles is given in Figure 10. One final question which we can attempt to answer is: What is the local conformation of the $S_{1}$ alkanone near the excited carbonyl group, i.e. is the $\mathrm{C}-\mathrm{CO}-\mathrm{C}$ group planar or puckered? We shall defer this question for the moment.

\section{PHOTOCYCLOADDITION OF ALKANONES TO ENOL ETHERS}

As in our discussion of the photocycloaddition of alkanones to nitriles, let us consider the question: Is the $S_{1}$ or $T_{1}$ state of the enol ether involved 


\section{CYCLOADDITION REACTIONS OF CARBONYL COMPOUNDS}

in the photocycloaddition? Indeed, the same type of experiments used to eliminate $S_{1}$ and $T_{1}$ of unsaturated nitriles as precursors to oxetanes, lead to the conclusion that neither $S_{1}$ nor $T_{1}$ of the enol ether adds to ground state alkanone $^{12}$ (see also Figure 6).

But how about $S_{1}$ and/or $T_{1}$ of the alkanone? In photocycloaddition of alkanones to enol ethers, our experimental data provide compelling arguments that both $S_{1}$ and $T_{1}$ add to the enol ether double bond.

We can immediately write Stern-Volmer equations analogous to 3 and 4 for the quenching of alkanone fluorescence by enol ethers and for formation of oxetane, as a function of enol ether.

$$
\begin{aligned}
& \phi_{F}^{0} / \phi_{F}=1+k_{q}^{F} \tau_{S}\left[\operatorname{ROR}^{\prime}\right] \\
& \left(1 / \phi_{0 \mathbf{x}}\right)=(1 / a)\left\{1+1 / k_{q}^{0 \mathbf{x}} \tau_{S}\left[\mathrm{ROR}^{\prime}\right]\right\}
\end{aligned}
$$

In this case although the observed kinetics follow equation 5 for fluorescence quenching, they are more complicated for oxetane formation. This result suggests that some intermediates in addition to $S_{1}$ can add to enol ethers and form oxetane. In order to elucidate this peculiar kinetic behaviour we studied the response of oxetane formation between acetone and 1-methoxybutene toward 1,3-pentadiene (a compound which quenches acetone triplets at the diffusion controlled rate ${ }^{31}$, but which is only a poor quencher of acetone singlets ${ }^{37}$ ). Strikingly ${ }^{12}$, at low concentrations of 1,3-pentadiene $(<0.05 \mathrm{M})$ and fixed concentration of 1-methoxybutene $(0.5 \mathrm{M})$ linear SternVolmer behaviour for oxetane formation was observed, then at higher concentrations of 1,3-pentadiene $(0.05-0.4 \mathrm{M})$ obvious curvature became apparent and at still higher concentrations of 1,3-pentadiene $(>0.4 \mathrm{M})$ no further quenching effect occurred. Since with 1-methoxybutene, the oxetane is formed with low, if any, stereospecificity and since cis-trans-isomerization accompanies oxetane formation, we can study the response of the degree of stereospecificity in oxetane formation and the efficiency of cis-transisomerization as a function of (a) 1-methoxybutene concentration and (b) 1,3-pentadiene concentration, since in this concentration region one of the precursors of oxetane formation is apparently totally quenched by 1,3pentadiene.

From analysis of the above kinetic data ${ }^{12}$ we conclude (see discussion below) that (a) both $S_{1}$ and $T_{1}$ of acetone are quenched by MB to produce a singlet and triplet intermediate(s) respectively. In addition, we suggest that both of the latter intermediates are capable of forming oxetane and effecting cis-trans-isomerization. Now the question arises: What is the nature of this intermediate? Some insight to help answer this important query is provided by the response of degree of stereospecificity and efficiency of cis-trans-isomerization toward (a) increasing concentration of 1methoxybutene and (b) quenching by 1,3-pentadiene. It is found that the degree of stereospecificity increases and the efficiency of cis-transisomerization decreases as the concentration of butene or diene increases. We conclude from these data that oxetane formation and cis-transisomerization are related reactions and that a (singlet) intermediate (whose formation is relatively favoured by high concentrations of butene and diene) 


\section{NICHOLAS J. TURRO}

collapses somewhat stereospecifically to oxetane ( $\sim 70$ per cent stereospecific starting from cis-1-methoxybutene, and $\sim 80$ per cent stereospecific starting from trans-1-methoxybutene) and is less efficient (roughly by a factor of ten) $)^{12}$ at effecting cis-trans-isomerization of the butene. A second (triplet) intermediate (whose formation is relatively favoured by low concentration of butene and diene) collapses essentially non-stereospecifically ( $\sim 4$ per cent stereospecificity starting from either cis-1-methoxybutene or trans-1methoxybutene).

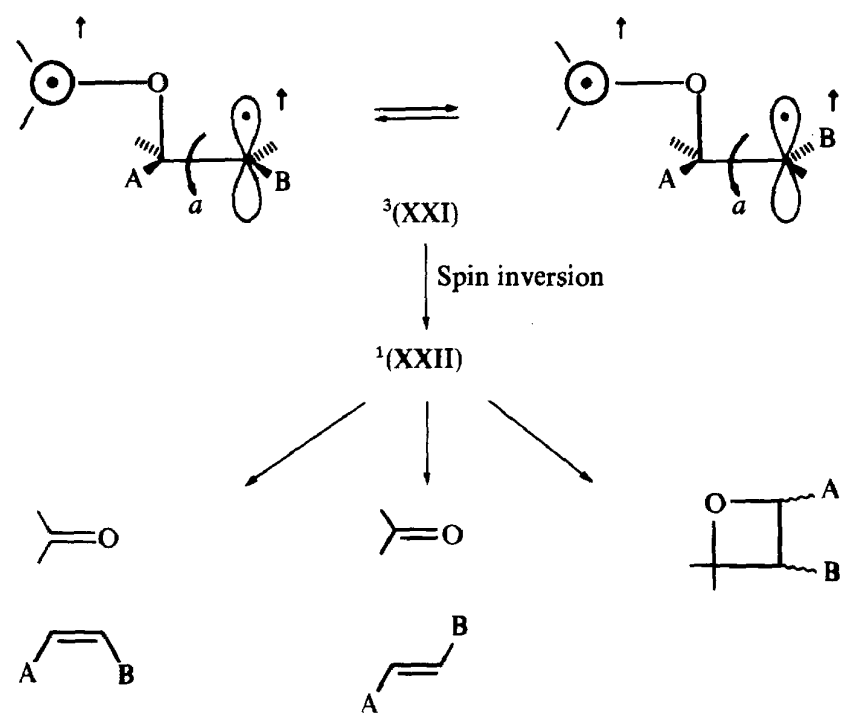

Figure 11. Configurational equilibration of the triplet biradical (XXI) occurs by rotation about bond $a$. Only transoid conformation of (XXI) is shown, but a family of interconverting cisoid and transoid conformations must be involved. (XXI) can only undergo two significant chemical processes: conformational interconversions and spin inversion to the singlet biradical (XXII). The latter is capable of undergoing (a) chemical bond rupture to yield a ground state alkanone and ethylene and (b) cyclization to an oxetane. Although internal disproportionation is a conceivable path for stabilization of (XXII) it does not appear to be significant in most cases studied to date

Finally, we also noted a close similarity of the percentage singlet cis $\rightarrow$ trans isomerization ( $\sim 7$ per cent) and the percentage singlet oxetane formation ( $\sim 10$ per cent) which further suggests correlation of the isomerization and oxetane-forming processes.

Do these intermediates possess biradical or bipolar characteristics? As mentioned earlier, a bipolar intermediate would be expected to show greater regiospecificity and solvent sensitivity than is actually found. The theoretical model for $S_{1}$ and $T_{1}$ suggests that the electrophilic $n$-orbital could attack the $\pi$ bond of the enol ether to form a biradical intermediate directly. This suggests an intermediate (Figure 11) which can collapse to oxetane, undergo configurational loss via bond rotations, and revert to alkanone and ethylene. 


\section{CYCLOADDITION REACTIONS OF CARBONYL COMPOUNDS}

This mechanism for cis-trans-isomerization was espoused by G. O. Schenck some time ago $^{38}$.

In order to explain these results it is sufficient to propose ${ }^{12}$ the biradical intermediates (XXI) and (XXII) (Figure 12). These structures are quite consistent with the expected products of $n$-orbital attack on the electron-rich enol ether $\pi$ system. The triplet biradical (XXI) which should be produced
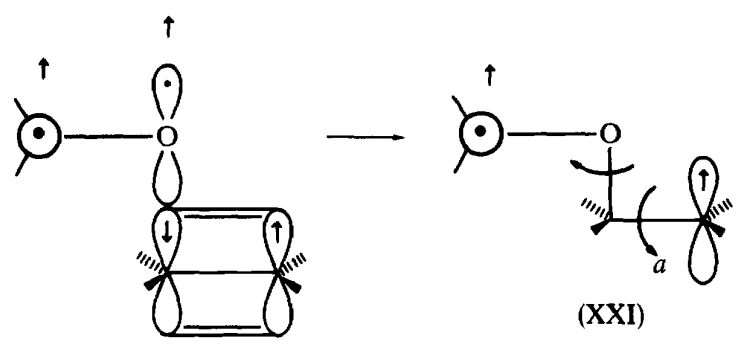

(XXI)
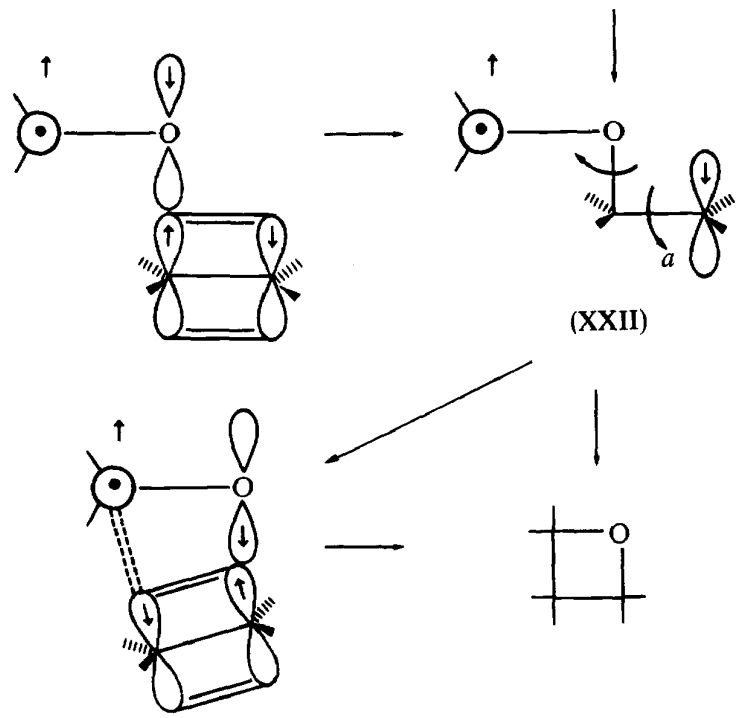

(XXII A)

Figure 12. Attack of $T_{1}$ on an ethylene to yield a triplet biradical (XXI), and attack of $S_{1}$ on an ethylene to yield a singlet biradical (XXII). The latter biradical is probably of lower energy since it is capable of stabilization by partition bonding of the spin paired free valences, but (XXI) is not capable of such stabilization. The cisoid transition (XXIIA) may also be capable of yielding

(XXII) or collapsing stereospecifically to oxetane. See text for further discussion

from $T_{1}$ and an enol ether cannot collapse to products (Figure 11) without a prior spin inversion ${ }^{39}$. As a result rotation about the bond $a$ can compete favourably with closure to oxetane and dissociation to ground state ketone and ethylene ${ }^{38}$. On the other hand, the singlet biradical (XXII) may be an example of a virtual biradical, i.e. a singlet biradical possessing one fully or nearly formed bond but for which there may be sufficient bonding between the spin-paired but space-separated free valence electrons so that rotation is 
NICHOLAS J. TURRO

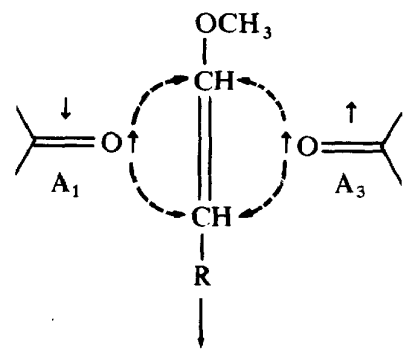

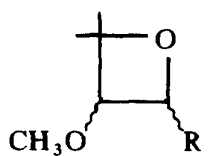

$c-3+t-3$

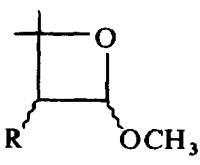

$c-2+t-2$

\begin{tabular}{|c|c|c|c|}
\hline \multicolumn{4}{|c|}{$[0.5 \mathrm{M}]$} \\
\hline $\begin{array}{l}t-\mathrm{MB} \\
t-3\end{array}$ & $\begin{array}{l}c-\mathbf{M B} \\
c-3\end{array}$ & $\begin{array}{l}c-\mathrm{MB} \\
c-2\end{array}$ & Conditions \\
\hline$\overline{c-3}$ & $\overline{t-3}$ & $\overline{t-2}$ & \\
\hline 1.2 & 1.4 & 0.88 & $\left(\mathrm{CH}_{3}\right)_{2} \mathrm{CO}$ as solvent \\
\hline 2.7 & 4.5 & 2.2 & $0.4 \mathrm{M}$ 1,3-pentadiene in $\left(\mathrm{CH}_{3}\right)_{2} \mathrm{CO}$ \\
\hline 1.04 & 0.97 & 0.65 & Extrapolated to zero [MB] \\
\hline
\end{tabular}

Figure 13. Regiospecificity of attack of $S_{1}$ and $T_{1}$ of acetone and cis- and trans-1-methoxybutene

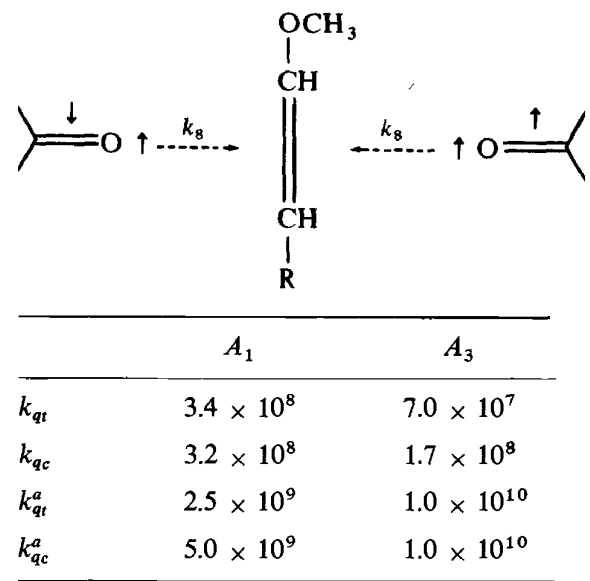

Figure 14. Rate constants for quenching of $S_{1}$ and $T_{1}$ of acetone by cis- and trans-1-methoxybutene. The values given for quenching of trans-1-methoxybutene $\left(k_{q t}\right)$ and cis-1-methoxybutene $\left(k_{q c}\right)$ are compared with the values for quenching of the $S_{1}$ and $T_{1}$ states of acetone by trans-dicyanoethylene $\left(k_{q t}^{a}\right)$ and cis-dicyanoethylene $\left(k_{q c}^{a}\right)$ 
hindered ${ }^{40}$ or slow, relative to closure or dissociation. It is also possible, of course, that $S_{1}$ and an enol ether form oxetane by a pair of simultaneous processes (a) a multistep non-stereospecific addition via a 'free' biradical and (b) a concerted four centre cycloaddition (Figure 12).

Two lines of evidence suggest that the concerted mechanism can be only of minor significance, at best: (a) the regiospecificity ${ }^{34}$ of attack of $S_{1}$ and $T_{1}$ on cis- or trans-1-methoxybutene are identical (Figure 13) within the experimental error, (b) the rate constants for attack of $S_{1}$ and $T_{1}$ on 1methoxybutene (Figure 14) are the same within (a rather broad) experimental error (suggesting similar transition states) ${ }^{42}$. It seems unlikely to us that a one-step and multi-step (the triplet is required to form oxetane in a multi-step process ${ }^{38}$ ) would show the same regiospecificity and kinetic behaviour. In conclusion, attack of $S_{1}$ of acetone on the double bond of enol ether probably yields a family of singlet biradical conformations and attack of $T_{1}$ yields a family of triplet biradical conformations. The chemistry of the latter family is probably limited in rate by spin inversion.

Table 4. Relative quenching rates of alkanone fluorescence by enol ethers ${ }^{\mathrm{a}}$

\begin{tabular}{cccc} 
Alkanone & cis- $\mathrm{C}_{2} \mathrm{H}_{5} \mathrm{OCH}=\mathrm{CHOC}_{2} \mathrm{H}_{5}$ & $\mathrm{CH}_{2}=\mathrm{C}\left(\mathrm{OC}_{2} \mathrm{H}_{5}\right)_{2}$ & $\mathrm{C}_{2} \mathrm{H}_{5} \mathrm{OCH}=\mathrm{CH}_{2}$ \\
\hline & $1.0^{\mathrm{b}}$ & 0.42 & $<0.032$ \\
\hline & $1.0^{\mathrm{c}}$ & & \\
\hline & & & \\
\hline
\end{tabular}

- Acetonitrile solutions.

b The absolute rate constant for fluorescence quenching is $2.0 \times 10^{9} \mathrm{M}^{-1} \mathrm{sec}^{-1}$

c The absolute rate constant for fluorescence quenching is $1.1 \times 10^{9} \mathrm{M}^{-1} \mathrm{sec}^{-1}$.

Table 4 summarizes the results of alkanone fluorescence quenching of some enol ethers, and adds further to the conclusion that $S_{1}$ acts as an electrophile in the transition state quenching.

\section{IS THERE A PRECURSOR TO THE BIRADICAL INTERMEDIATE?}

The lack of regiospecificity of oxetane formation from alkanones and enol ethers is of special interest. One might simply say that the transition state for oxetane formation in these cases looks like a high energy non-selective intermediate. However, the rate constants for fluorescence quenching suggest that the transition state for deactivation of alkanone singlets is quite sensitive to both alkanone structure and to enol ether structure.

Data in the literature suggest that the biradical intermediate is at best a mediocre model for predicting the regiospecificity of addition of aryl ketone triplets to alkenes 5,6 . Furthermore, both rate studies ${ }^{41,42}$ and isotope effects $^{43,44}$ suggest that the interaction by which an aryl ketope triplet is 
quenched by an alkene does not lead directly to a biradical, although strong evidence exists for a biradical intermediate prior to oxetane formation, i.e. photocycloaddition of benzophenone triplets to cis- and trans-butene is non-stereospecific ${ }^{3}$.

What lines of evidence require or support a precursor to the biradical in excited alkanones and enol ethers? So far, we can only produce indirect evidence that the transition state for quenching of excited alkanones is not identical to the transition state for formation of the biradical: (a) the poor to modest regioselectivity, especially with 1,1-diethoxyethylene, which would be expected to yield a much higher ratio of 3- to 2-alkoxyoxetane than is found; (b) the increase in the fluorescence quenching rate constant as one goes from ethyl vinyl ether to 1,1-diethoxyethylene amounts to a factor greater than ten, yet the ratio of 3- to 2-oxetane is within the experimental error for these two enol ethers (Table 2).

An important complicating factor in understanding the meaning of the low regiospecificity is the possibility of reversibility of biradical formation, since one of the two possible biradicals formed by addition of an excited alkanone may collapse more efficiently to ground state ketone and ethylene than to oxetane.

Although we have no particular bias for the structure of the speculative precursor to the biradical, we feel that the major interaction of the alkanone $S_{1}$ and $T_{1}$ states (which directly leads to quenching) is probably associated with the entire $\pi$ cloud of the enol ether, rather than more markedly with one particular unsaturated carbon atom, and that a tight radical cation-radical ion pair (which preserves spin) or some structurally related species precedes the singlet and triplet biradicals.

\section{FURTHER STRUCTURE/REACTIVITY TESTS OF PHOTOCYCLOADDITION VIA ALKANONE SINGLET STATES}

Up to this point we have seen that two models are required to explain the interaction of alkanone $S_{1}$ states with electron-poor and electron-rich ethylenes. Since we have seen that the rate of alkanone fluorescence quenching by unsaturated nitriles and enol ethers can be correlated with the rates of oxetane formation, we can use equations 3 and 5 to provide useful information on the relationship of alkanone structure to the rate of quenching by a standard nitrile or standard enol ether. Such studies have proved to be extremely revealing. Table 5 lists the relative and absolute rate constants for fluorescence quenching of a series of norcamphor derivatives by transdicyanoethylene ( $t$-DCE) and cis-1,2-diethoxyethylene ( $c$-DEE). For these rigid structures which do not possess sufficient flexibility (cf. monocyclic ketones, vide infra) to minimize steric effects, we shall assume that the only major departure from ground state geometry for $S_{1}$ must occur locally about the $\mathrm{C}-\mathrm{CO}-\mathrm{C}$ group. Inspection of the data in Table 5 reveals that the response of $k_{q}^{F}$ for $t$-DCE shows a strong resemblance to the reactivity pattern for attack of nucleophiles on ground state ${ }^{45}$ bicyclo-[2,2,1]-2-heptanones. 


\section{CYCLOADDITION REACTIONS OF CARBONYL COMPOUNDS}

Thus, we can conclude that exo attack on $S_{1}$ by $t$-DCE is favoured for (XXIII), while (XXIV), which has the approach to exo attack hindered by the 7-methyl substituents, is quenched at a much slower rate. In striking contrast, $c$-DEE quenches the $S_{1}$ states of (XXIII) and (XXIV) at essentially the same rate! Furthermore (XXV), which is still moderately quenched by $t$-DCE, is only poorly quenched by $c$-DEE. We interpret these data as compelling evidence for the occurrence of two distinct quenching mechanisms. In one

Table 5. Relative fluorescence quenching rates for $[2,2,1]$ bicycloheptanones by trans-1,2-dicyanoethylene and cis1,2-diethoxyethylene ${ }^{\mathrm{a}}$

Alkanone

- Acetonitrile solutions.

b Absolute rate constant for fluorescence quenching is $6.0 \times 10^{9} \mathrm{M}^{-1} \mathrm{sec}^{-1}$.

- Rates are based on the assumption that $\tau_{s}$ for (XXIII), (XXIV) and (XXV) are approximately equal.

d Absolute rate constant for fluorescence quenching is $1.4 \times 10^{9} \mathrm{M}^{-1} \mathrm{sec}^{-1}$.

case (Figure 15) quenching of $S_{1}$ occurs when the electron-poor double bond of $t$-DCE interacts (preferentially) with the nucleophilic $\pi$ faces of the excited carbonyl group. In the other case, the quenching of $S_{1}$ occurs when the electron-rich double bond of $c$-DEE interacts (preferentially) with the electrophilic half-filled $n$ orbital/of the excited carbonyl group. These data also imply that the $\mathrm{C}-\mathrm{CO}-\mathrm{C}$ function of $S_{1}$ is not severely non-planar, and confirm the position that concerted cycloaddition via a four-centred transition state (similar to that for $S_{1}$ and nitriles) does not occur to any significant extent with $S_{1}$ and enol ethers. 

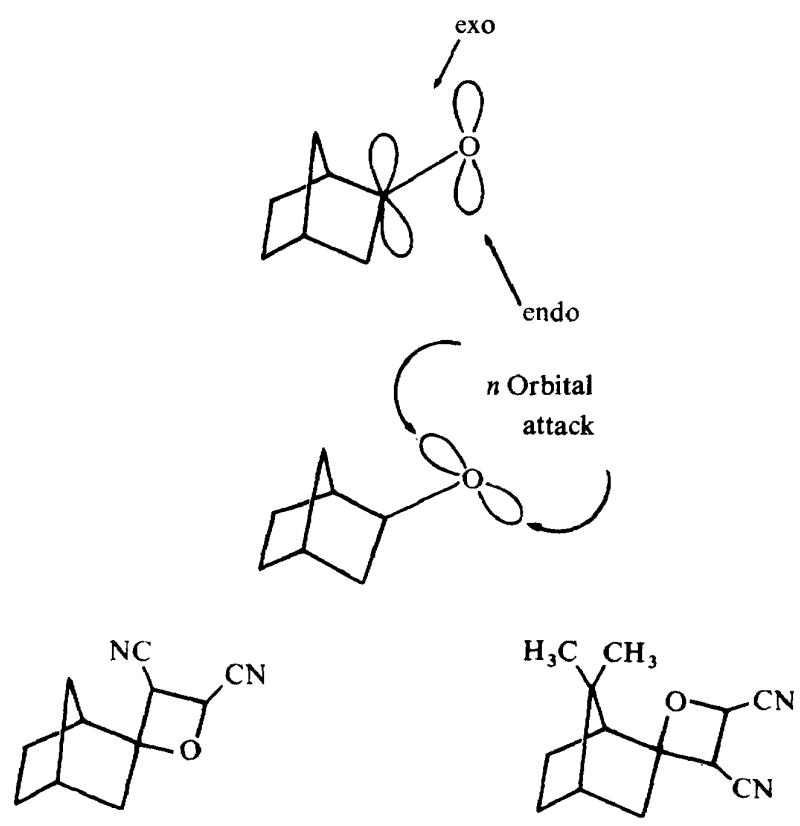

Figure 15. Different types of attack on the carbonyl of $S_{1}$ of bicyclo-[2,2,1]-heptanones: (a) exo attack is favoured when an $\alpha, \beta$-unsaturated nitrile is quencher, but 7,7-dimethyl substituents sterically slow down exo attack and may force quenching by attack on the endo face; (b) 'edge' or $n$ orbital attack is favoured when an enol ether is quencher. The tentative structures for photocycloaddition of trans-1,2-dicyanoethylene to norcamphor and 7,7-dimethylnorcamphor are shown

\section{CONFORMATIONAL ANALYSES OF THE EXCITED SINGLET STATES OF CYCLIC ALKANONES}

The foregoing discussion has provided strong evidence for the proposition that excited singlet alkanones are attacked by unsaturated nitriles above or below the nucleophilic carbonyl $\pi^{*}$ faces, while enol ethers attack the electrophilic half $n$ orbital on either side of the carbonyl oxygen. We are now in a position to apply this mechanism to other systems, and on the basis of the experimental results we can attempt to make conclusions about the excited state conformation about the $\mathrm{C}-\mathrm{CO}-\mathrm{C}$ group and, for flexible cyclic systems, the conformation of the hydrocarbon-chain of the excited state. Table 6 gives the results of fluorescence quenching of some alkylated cyclohexanones by trans-1,2-dicyanoethylene and cis-1,2-diethoxyethylene ${ }^{46}$. Table 7 summarizes similar data for fluorescence quenching of a series of monocyclic ketones.

Examination of the data in Table 6 suggests that $t$-DCE attacks the axial face of the $S_{1}$ alkanone (Figure 16) preferentially with attack on the equitorial face being less favoured. The response of $k_{q}^{F}$ to steric factors is reminescent of 'steric approach control' (attack from the least hindered face of the 
carbonyl) of the rate of attack of nucleophilic metal hydrides on ground state cyclohexanones ${ }^{47}$. The identity of quenching rate for (XXVI) and (XXVII) suggests that the chair form of cyclohexanones, and not their boat forms, are the major species active in the quenching step.

Table 6. Relative rates of fluorescence quenching of some alkyl cyclohexanones $^{\mathrm{a}}$

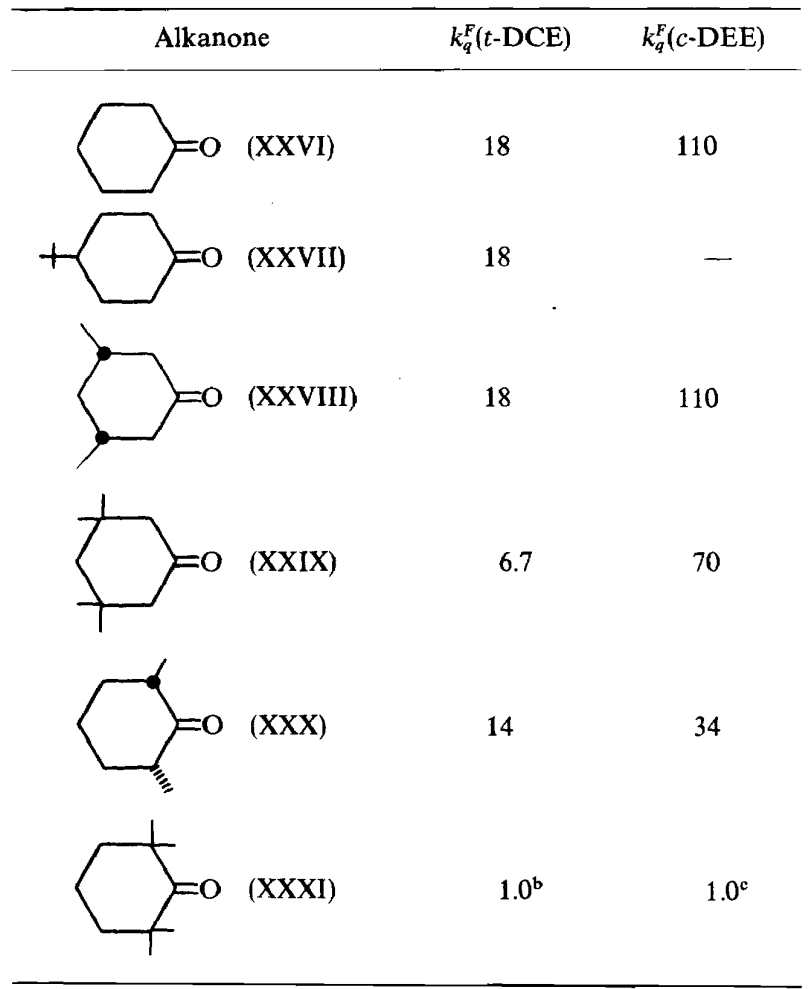

Acetonitrile solutions

b Absolute rate constant for fluorescence quenching is $1.8 \times 10^{8} \mathrm{M}^{-1} \mathrm{sec}^{-1}$.

- Absolute rate constant for fluorescence quenching is $2.0 \times 10^{7} \mathrm{M}^{-1} \mathrm{sec}^{-1}$

A very recent and potentially exciting area of research may reside in attempting to unravel the conformational behaviour of medium and large ring cyclic ketones (Table 7$)^{48}$. Data from a number of different types of measurements have established that cyclic ketones, of size near ten ring atoms, exhibit peculiar chemical properties ${ }^{49}$. There is evidence that, relative to the smaller and larger ring homologues, cyclododecanone may possess confirmations ${ }^{50}$ which place the carbonyl group 'inside' (Figure 17.) the ring, in order to minimize non-bonded interactions. With these considerations in mind, we note that the cyclic $\mathrm{C}_{10}$ ketone appears to be poorly quenched by $t$-DCE and $c$-DEE relative to its lower and higher homologues. 
NICHOLAS J. TURRO

Table 7. Relative rates of fluorescence quenching of some monocyclic alkanones $^{\mathbf{a}}$

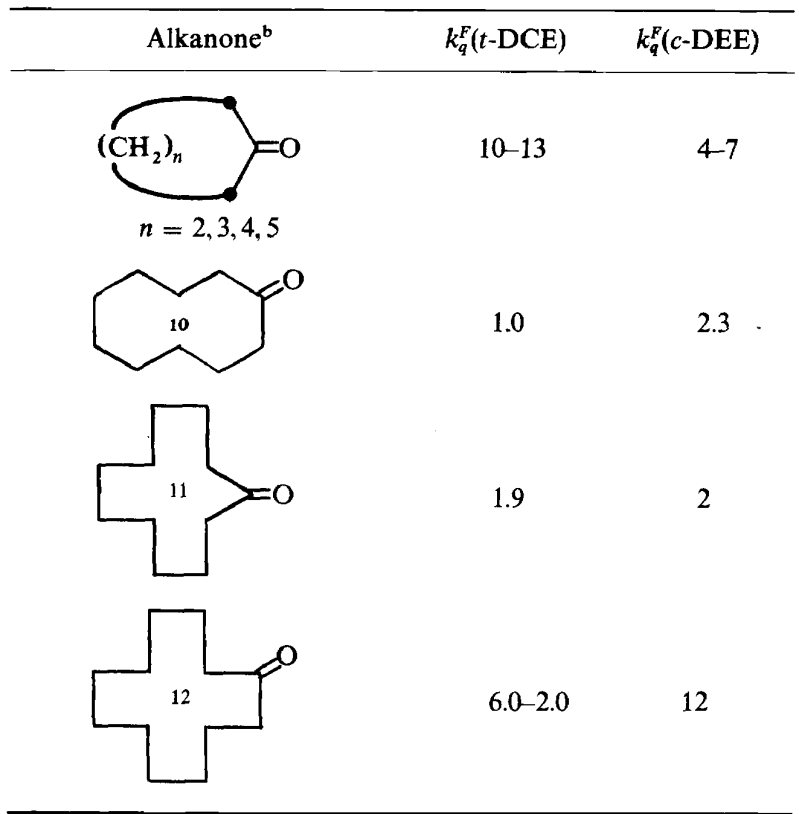

* Acetonitrile solutions.

- The values in the table are uncorrected for differences in singlet lifetimes.
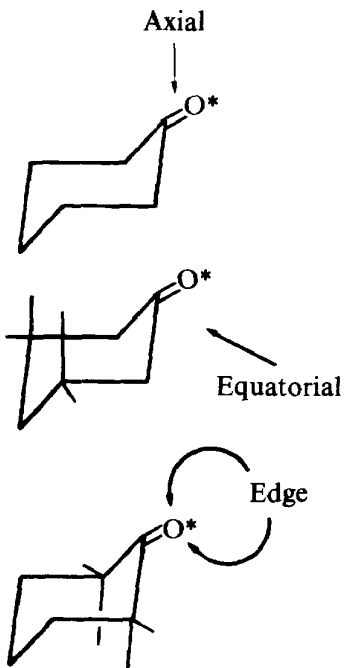

Figure 16. Axial attack on $S_{1}$ is favoured over equatorial attack by $\alpha, \beta$-unsaturated nitrile for flexible, relatively unhindered cyclohexanones. Axial substituents $\beta$ to the carbonyl sterically interfere with axial attack and may force quenching to occur predominantly by attack on the equatorial face of the carbonyl. Edge attack on the $n$ orbital is favoured for quenching of $S_{1}$ by enol ethers, and is much more sensitive to equatorial 2,6-substituents than 3,5-substituents 


\section{CYCLOADDITION REACTIONS OF CARBONYL COMPOUNDS}

The spread in values for $k_{q}^{F}$ of cyclododecanone by $t$-DCE is the result of non-linear Stern-Volmer quenching, a result consistent with $t$ wo quenchable $S_{1}$ states! Perhaps two different fluorescent conformers are being quenched. Further studies along these lines should be quite fruitful in expanding our rather feeble and limited present understanding of the conformation of excited states.

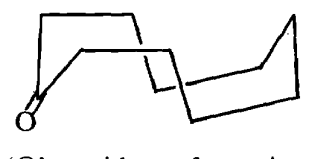

'O' outside conformation

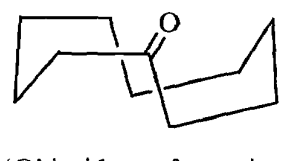

' $\mathrm{O}$ ' inside conformation

Figure 17. Pictorial description of the ' $O$ ' outside and ' $O$ ' inside conformations of cyclodecanone

It should also be noted that since the $C_{5}$ to $C_{8}$ inclusive monocyclic alkanones show very similar quenching behaviour, ' $I$ ' strain does not appear to be important in the developing transition state for the quenching step, i.e. the transition state appears early along the reaction profile, a conclusion consistent with our earlier discussion of 'steric approach control' of the quenching of $[2,2,2]$-bicycloheptanones and alkylcyclohexanones.

All in all, these results suggest that although the excited $\mathrm{C}-\mathrm{CO}-\mathrm{C}$ function may be somewhat puckered, a ground state model of a planar $\mathrm{C}-\mathrm{CO}-\mathrm{C}$ group is sufficient for many predictive purposes concerning the structure/reactivity relationships of oxetane formation and fluorescence quenching of alkanones by ethylenes.

\section{ACKNOWLEDGEMENT}

The research and ideas reported herein are the cooperative efforts and results of the patience, experimental skill and creativity of a number of collaborators, students and post-doctoral associates. In particular, it gives me great pleasure to acknowledge the considerable experimental and intellectual contributions of Dr Peter A. Wriede, Dr David M. Pond and Prof. J. Christopher Dalton, as well as more recent and substantial contributions of Mr Mark Niemczyk, Mr George Farrington and Mr Ching Lee. This work was generously supported by the Air Force Office of Scientific Research.

\section{REFERENCES}

1 E. Paterno and G. Chieffi, Gazz. Chim. Ital. 39, 341 (1909).

2 G. Buchi, C. G. Inman and E. S. Lipinsky, J. Am. Chem. Soc. 76, 4327 (1954);

G. Buchi, J. T. Kofron, E. Koller and D. Rosenthal, J. Am. Chem. Soc. 78, 876 (1956). 
${ }^{3}$ D. R. Arnold, R. L. Hinman and A. H. Glick, Tetrahedron Letters, 1425 (1964).

4 N. C. Yang, Pure Appl. Chem. 9, 591 (1964);

N. C. Yang, M. Nussim, M. J. Jorgenson and S. Murov, Tetrahedron Letters, 3657 (1964).

5 D. R. Arnold, Advanc. Photochem. 6, 301 (1968).

${ }^{6}$ L. L. Muller and J. Hamer, 1,2-Cycloaddition Reactions, p 111. Interscience: New York (1967).

7 N. J. Turro, P. Wriede, J. C. Dalton, D. Arnold and A. Glick, J. Am. Chem. Soc. 89, 3950 (1967);

J. J. Beereboom and M. S. von Wittenau, J. Org. Chem. 30, 1231 (1965).

8 N. J. Turro, P. A. Wriede and J. C. Dalton, J. Am. Chem. Soc. 90,3274 (1968).

9 N. J. Turro and P. A. Wriede, J. Am. Chem. Soc. 90,6863 (1968).

10 N. J. Turro and P. A. Wriede, J. Org. Chem. 34, 3562 (1969).

11 J. C. Dalton, D. M. Pond and N. J. Turro, J. Am. Chem. Soc. 92, 2173 (1970).

12 N. J. Turro and P. A. Wriede, J. Am. Chem. Soc. 92, 320 (1970).

13 J. C. Dalton, P. A. Wriede and N. J. Turro, J. Am. Chem. Soc. 92, 1318 (1970).

14 N. J. Turro, J. C. Dalton, G. Farrington, Mark Niemczyk and D. M. Pond, J. Am. Chem. Soc. 92, 6978 (1970).

15 J. C. Dalton and N. J. Turro, Ann. Rev. Phys. Chem. 21,499 (1970).

16 N. J. Turro, Molecular Photochemistry, Ch. 6. W. A. Benjamin: New York (1965).

17 R. F. Borkman and D. R. Kearns, J. Am. Chem. Soc. 88, 3467 (1966).

${ }^{18}$ M. Kasha in Light and Life, p 31; W. D. McElroy and B. Glass, eds., Johns Hopkins: Baltimore, Maryland (1961);

G. W. Wheland, Resonance in Organic Chemistry, p 283. Wiley: New York (1955);

H. E. Zimmerman, Advanc. Photochem. 1, 183 (1963).

19 J. A. Barltrop and H. A. J. Carless, Tetrahedron Letters, 3901 (1968).

20 D. R. Arnold and A. H. Glick, Chem. Commun. 813 (1966).

21 H. Gotthardt, R. Steinmetz and G. S. Hammond, J. Org. Chem. 33, 2774 (1968)

22 S. H. Schroeter, Chem. Commun. 12 (1969).

23 S. H. Schroeter and C. M. Orlando Jr, J. Org. Chem. 34, 1181 (1969).

24 S. Toki and H. Sakurai, Bull. Chem. Soc. Japan, 40, 2885 (1967).

25 P. A. Wriede, Columbia University, unpublished results. In the case of 1,1-diethoxyethylene a material thought to be a dimer could be isolated in low yield, but the material facilely decomposed to 1,1-diethoxyethylene.

26 P. A. Wriede, Columbia University, unpublished results. See also ref. 23.

27 J. Sauer, Angew. Chem. Intern. Ed. Eng. 6, 16 (1967);

R. Huisgen, R. Graskey and J. Sauer, in Chemistry in Alkenes, p 739. S. Patai, ed., Interscience: New York (1964).

28 S. Hosaka and S. Wakamatsu, Tetrahedron Letters, 219 (1968).

${ }^{29}$ M. Herberhold and G. S. Hammond. Ber. Bunsenges. Phys. Chem. 72, 309 (1968).

30 D. M. Gale, J. Org. Chem. 35, 970 (1970);

R. S. H. Liu and D. M. Gale, J. Am. Chem. Soc. 90, 1897 (1968).

31 P. J. Wagner, J. Am. Chem. Soc. 89, 2503 (1967); J. Am. Chem. Soc. 88, 5672 (1966).

32 A number of examples of singlet donor to triplet acceptor electronics excitation transfer are known: V. L. Ermolaev and E. B. Sveshnikova, Opt. Spectr. 28, 324 (1970) and references therein.

33 Exciplex mechanisms for quenching of fluorescence have been proposed previously: L. M. Stephenson and G. S. Hammond, Angew. Chem. Intern. Ed. Eng. 8, 261 (1969);

L. M. Stephenson and G. S. Hammond, Pure Appl. Chem. 16, 125 (1968).

34 Since the efficiency of cycloaddition is not high, one must worry about reversibility of intermediate formation when trying to correlate regiospecificity with kinetic selectivity.

35 T. Förster, Angew. Chem. Intern. Ed. Eng. 8, 333 (1969); exciplexes are molecular aggregates which are stable only in electronically excited states but not in ground states. In many cases a good correlation exists between donor ionization potential and acceptor electron affinity and exciplex stability.

36 A. N. Nesmeyanov, M. I. Rybinskaya and L. V. Rybin, Russ. Chem. Rev. 36, 453 (1967).

${ }^{37}$ F. S. Wettack, G. D. Renkes, M. G. Rockley, N. J. Turro and J. C. Dalton, J. Am. Chem. Soc. 92, 1793 (1970).

38 G. O. Schenck and R. Steinmetz, Bull. Soc. Chim. Belges, 71, 781 (1962).

39 It is likely that spin will be conserved in an elementary chemical step.

${ }^{40}$ It is entirely conceivable that $\sigma$ bonds adjacent to a free radical centre interact strongly with 


\section{CYCLOADDITION REACTIONS OF CARBONYL COMPOUNDS}

the partially vacant orbital and cause hindrance to the normally 'free' rotation about single bonds.

41 N. C. Yang, Photochem. Photobiol. 7, 767 (1968).

42 N. C. Yang, J. I. Cohen and A. Shani, J. Am. Chem. Soc. 90, 3264 (1968);

N. C. Yang, R. Loeschen and D. Mitchell, J. Am. Chem. Soc. 89, 5465 (1967).

43 R. A. Caldwell, J. Am. Chem. Soc. 92,1439 (1970).

44 R. A. Caldwell and S. P. James, J. Am. Chem. Soc. 91,5184 (1969).

45 H. C. Brown and J. Muzzio, J. Am. Chem. Soc. 88, 2811 (1966);

H. C. Brown and J. H. Kawakami, J. Am. Chem. Soc. 92, 201 (1970).

46 D. Pond, M. Niemczyk, G. Farrington and J. C. Dalton, Columbia University, unpublished results.

${ }^{47}$ E. L. Eliel and Y. Senda, Tetrahedron, 26, 2411 (1970) and references therein:

48 N. J. Turro, M. Niemczyk and D. M. Pond, Molec. Photochem. 2, 345 (1970).

49 V. Prelog, J. Chem. Soc. $420(1950)$.

so V. Prelog, Pure Appl. Chem. 6, 545 (1963). 